\title{
Schur Coefficients of the Integral Form Macdonald Polynomials
}

\author{
Meesue YOO \\ Korea Institute for Advanced Study \\ (Communicated by N. Suwa)
}

\begin{abstract}
In this paper, we consider the combinatorial formula for the Schur coefficients of the integral form of the Macdonald polynomials. As an attempt to prove Haglund's conjecture that $\left\langle\frac{J_{\mu}\left(X ; q, q^{k}\right)}{(1-q)^{|\mu|}}, s_{\lambda}(X)\right\rangle \in \mathbf{N}[q]$, we have found explicit combinatorial formulas for the Schur coefficients in one row case, two column case and certain hook shape cases [Yoo12]. A result of Egge-Loehr-Warrington [ELW10] gives a combinatorial way of getting Schur expansion of symmetric functions when the expansion of the function in terms of Gessel's fundamental quasi symmetric functions is known. We apply this result to the combinatorial formula for the integral form Macdonald polynomials of Haglund [Hag08] in quasi symmetric functions to prove the Haglund's conjecture in more general cases.
\end{abstract}

\section{Introduction}

In 1988, I. G. Macdonald [Mac95] introduced a remarkable new basis for the space of symmetric functions. The elements of this basis are denoted $P_{\mu}(X ; q, t)$ where $\mu$ is a partition, $X=\left(x_{1}, x_{2}, \ldots\right)$, and $q, t$ are two free parameters. The $P_{\mu}(X ; q, t)$ 's, which are now called Macdonald polynomials, specialize to many of the well-known bases for the symmetric functions by suitable choices of the parameters $q$ and $t$. They were immediately hailed as a breakthrough in symmetric function theory as well as special functions, as they contained most of the previously studied families of symmetric functions as special cases, and yet satisfied many interesting properties, such as a multivariate orthogonality relation. Upon the introduction, Macdonald also defined the integral forms $J_{\mu}(X ; q, t)$ of $P_{\mu}$ by multiplying certain polynomial of $q$ and $t$, and conjectured that they can be expanded in terms of modified Schur functions $s_{\lambda}[X(1-t)]$ with coefficients in $\mathbf{N}[q, t]$. This is known as the Macdonald positivity conjecture and it has been proved by Haiman [Hai01] algebraically, and by Assaf [Ass07] combinatorially.

On the other hand, Haglund noticed and conjectured that for any $k \in \mathbf{N}$,

$$
\left\langle\frac{J_{\mu}\left(X ; q, q^{k}\right)}{(1-q)^{|\mu|}}, s_{\lambda}(X)\right\rangle \in \mathbf{N}[q]
$$

Received November 26, 2013; revised June 18, 2014

1991 Mathematics Subject Classification: 05A10

Key words and phrases: Schur coefficients, Macdonald polynomials, ELW-method 
and the conjecture has been verified by the author [Yoo12] in one-row case, two column case and certain hook shape cases by finding explicit combinatorial descriptions for the Schur coefficients. In this paper, to further investigate Haglund's conjecture, we apply the combinatorial method of Egge-Loehr-Warrington [ELW10] to get the Schur coefficients of $J_{\mu}(X ; q, t)$, given the combinatorial formula for $J_{\mu}(X ; q, t)$ in terms of the fundamental quasisymmetric functions [Hag08].

\section{Background}

For a permutation $\sigma \in S_{n}$, let $\operatorname{Des}(\sigma)$ denote the set of $i \in\{1,2, \ldots, n-1\}$ such that $i+1$ appears to the left of $i$ in $\sigma$. If $\operatorname{Des}(\sigma)=\left\{i_{1}<i_{2}<\cdots<i_{k}\right\}$, then we define $\operatorname{Des}^{\prime}(\sigma)$ to be the corresponding composition $\left(i_{1}, i_{2}-i_{1}, \ldots, n-i_{k}\right) \vDash n$. Given a partition $\lambda$, a standard Young tableau of shape $\lambda$ is a bijection $T: \operatorname{dg}(\lambda) \rightarrow\{1,2, \ldots, n\}$ for which $T$ values are increasing along the rows and up the columns of $\operatorname{dg}(\lambda)$, where $\operatorname{dg}(\lambda)$ is the left-south justified Young diagram of $\lambda$. We let $\operatorname{SYT}(\lambda)$ denote the set of standard Young tableaux of shape $\lambda$. Given $T \in \operatorname{SYT}(\lambda)$, define the reading word $\operatorname{rw}(T)$ by reading the values of $T$ along the rows from left to right, starting from top to bottom. Then define $\operatorname{Des}(T)$ and $\operatorname{Des}^{\prime}(T)$ to be $\operatorname{Des}(\operatorname{rw}(T))$ and $\operatorname{Des}^{\prime}(\operatorname{rw}(T))$. Note that the Schur function can be expanded in terms of the fundamental quasisymmetric functions as a sum over the set of standard Young tableaux.

THEOREM 2.1 [Ges84, Sta99].

$$
s_{\lambda}=\sum_{T \in S Y T(\lambda)} F_{D e s^{\prime}(T)} .
$$

2.1. Schur versus quasisymmetric expansion. A skew diagram $\lambda / \mu$ is a rim-hook of $\lambda$ if $\lambda / \mu$ does not contain any $2 \times 2$ subdiagram and any two consecutive cells of $\lambda / \mu$ share an edge. A rim-hook is special if it starts from the cell in the first column. The number of rows of a rim hook $H$ is referred to as its height, denoted by $\mathrm{ht}(H)$. The sign of a rim hook $H$ is defined to be $(-1)^{\mathrm{ht}(H)-1}$. A special rim-hook tableau $S$ of shape $\lambda$ and content $\alpha$ is a partition of the diagram of $\lambda$ using special rim-hooks such that the length of the $i$-th rim-hook from the bottom is $\alpha_{i}$. The sign of $S$ is the product of the signs of the rim hooks of $S$. For more details about rim-hook tableaux, see [ER90].
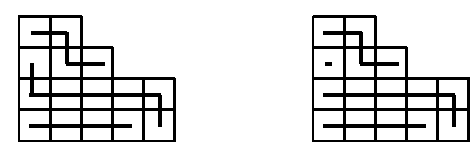

FIGURE 1. Examples of special rim hook tableaux of shape $\lambda=(5,5,3,2)$ 
Egge-Loehr-Warrington [ELW10] gives a combinatorial description of Schur coefficients, given a fundamental quasisymmetric expansion of any symmetric functions.

THEOREM 2.2 [ELW10, Theorem 11]. Suppose $\mathbf{F}$ is a field, and we have a symmetric function

$$
f=\sum_{\lambda \vdash n} c_{\lambda} s_{\lambda}=\sum_{\alpha \models n} d_{\alpha} F_{\alpha} \quad\left(c_{\lambda}, d_{\alpha} \in \mathbf{F}\right) .
$$

Then we have

$$
c_{\lambda}=\sum_{\alpha \models n} d_{\alpha} K_{n}^{*}(\alpha, \lambda)
$$

for all $\lambda \vdash n$, where

$$
K_{n}^{*}(\alpha, \lambda)=\sum_{\beta \text { finer than } \alpha} K_{n}^{\prime}(\beta, \lambda),
$$

and $K_{n}^{\prime}$ is a right inverse of the Kostka matrix $K_{n}$ with entries $K_{n}^{\prime}(\alpha, \lambda)$, the sum of the signs of the special rim-hook tableaux of shape $\lambda$ and content $\alpha$.

If each rim-hook contains exactly one cell in the first column of the Ferrers diagram of $\lambda$, then we say that the rim-hook tableau $S$ of shape $\lambda$ and content $\alpha$ (or equivalently, $(\alpha, \lambda)$ ) is flat. Then we can simplify the description of $K_{n}^{*}(\alpha, \lambda)$ even more.

THEOREM 2.3 [ELW10, Theorem 15]. Let $\alpha \models n, \lambda \vdash n$. If $(\alpha, \lambda)$ is flat, then $K_{n}^{*}(\alpha, \lambda)=K_{n}^{\prime}(\alpha, \lambda)= \pm 1$. Otherwise, $K_{n}^{*}(\alpha, \lambda)=0$. In particular, $K_{n}^{*}(\alpha, \lambda)=\chi(\alpha=\lambda)$ when $\lambda$ is a hook.

2.2. Quasisymmetric expansion of $J_{\mu}(X ; q, t)$. Given a diagram of a partition $\mu$, three cells $u, v, w \in \mu$ are said to form a triple if they are situated as shown below,

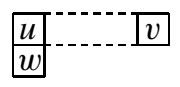

including the case when $u$ and $v$ are in the first row. Given a standard filling $\sigma$ of $\mu$, we define an orientation on such a triple by starting at the square containing the smallest element of $\sigma$, and going in a circular motion, towards the next largest and ending at the largest element. We say that the triple is an inversion triple if this circular motion is counterclockwise, and coinversion triple if the circular motion is clockwise. For the cases when $u$ and $v$ are in the first row, we put $-\infty$ 's below the cells in the first row. Given a triple $u, v, w$ in $\mu$, we define the middle square of the triple, with respect to $\sigma$, to be the square containing the middle number of the three $\{\sigma(u), \sigma(v), \sigma(w)\}$. If $u$ and $v$ are in the first row, then we let the middle square be $u$ if $\sigma(u)<\sigma(v)$, and $v$ otherwise. Then define $\operatorname{coinv}_{s}(\sigma, \mu)$ to be the number of coinversion triples for which $s$ is the middle square, and $\operatorname{inv}_{s}(\sigma, \mu)$ to be the number of inversion triples for which $s$ is the middle square. 
For a given filling $\sigma$ of $\mu$ and $s \in \mu$, let

$$
\begin{aligned}
\operatorname{nondes}_{s}(\sigma, \mu) & = \begin{cases}\operatorname{leg}(s)+1, & \text { if } \sigma(\operatorname{South}(s)) \geq \sigma(s) \text { and South }(s) \in \mu, \\
0, & \text { otherwise }\end{cases} \\
\operatorname{maj}_{s}(\sigma, \mu) & = \begin{cases}\operatorname{leg}(s), & \text { if } \sigma(\operatorname{North}(s))>\sigma(s) \text { (i.e., descent at North) } \\
0, & \text { otherwise, }\end{cases}
\end{aligned}
$$

where $\operatorname{South}(s)$ denotes the square below $s$ and $\operatorname{North}(s)$ denotes the square above $s$.

We recall that Haglund [Hag08, Corollary A.12.1] derived the quasisymmetric expansion of the integral form Macdonald polynomials from the combinatorial formula for the Macdonald polynomials of Haglund-Haiman-Loehr [HHL05].

THEOREM 2.4 [Hag08].

$$
J_{\mu}(X ; q, t)=\sum_{\substack{w \in S_{n} \\ \text { primary }}} D_{(w, \mu)}(q, t) F_{\operatorname{Des}^{\prime}(w)}(X),
$$

where primary implies that $i$ can occur in the bottom $i$ rows, for $1 \leq i \leq n$, and

$$
D_{(w, \mu)}(q, t)=\prod_{s \in \mu}\left(q^{i n v_{s}(w, \mu)} t^{\text {nondes }_{s}(w, \mu)}-q^{\operatorname{coinv}_{s}(w, \mu)} t^{1+m a j_{s}(w, \mu)}\right),
$$

and we identify a permutation $w$ with the standard filling of $\mu$ whose reading word is $w$.

Then by combining Theorem 2.2 and Theorem 2.3, we can get the Schur coefficients of $J_{\mu}(X ; q, t)$.

$$
\begin{aligned}
J_{\mu}(X ; q, t) & =\sum_{\substack{w \in S_{n} \\
\text { primary }}} D_{(w, \mu)}(q, t) F_{\operatorname{Des}^{\prime}(w)}(X) \\
& =\sum_{\lambda \vdash n}\left(\sum_{\substack{w \in S_{n} \\
\text { primary }}} D_{(w, \mu)}(q, t) K_{n}^{\prime}\left(\operatorname{Des}^{\prime}(w), \lambda\right)\right) s_{\lambda},
\end{aligned}
$$

where $K_{n}^{\prime}\left(\operatorname{Des}^{\prime}(w), \lambda\right)$ is \pm 1 up to the sign of the special rim-hook tableau. In this paper, we compute specific Schur coefficients of certain cases by using the combinatorial description of $K_{n}^{\prime}\left(\operatorname{Des}^{\prime}(w), \lambda\right)$.

REMARK 2.5. Note the following combinatorial formula for $J_{\mu}(X ; q, t)$.

THEOREM 2.6 [HHL05].

$$
J_{\mu}(X ; q, t)=\sum_{\begin{array}{c}
\text { nonattackingfillings } \\
\sigma \text { of } \mu^{\prime}
\end{array}} X^{\sigma} q^{\operatorname{maj}\left(\sigma, \mu^{\prime}\right)} t^{\operatorname{coinv}\left(\sigma, \mu^{\prime}\right)}
$$




$$
\times \prod_{\substack{u \in \mu^{\prime} \\ \sigma(u)=\sigma(\operatorname{South}(u))}}\left(1-q^{\operatorname{leg}(u)+1} t^{\operatorname{arm}(u)+1}\right) \prod_{\substack{u \in \mu^{\prime} \\ \sigma(u) \neq \sigma(\operatorname{South}(u))}}(1-t) .
$$

If we set $t=q^{k}$ and divide by $(1-q)^{|\mu|}$, then we get

$$
\begin{aligned}
\frac{J_{\mu}\left(X ; q, q^{k}\right)}{(1-q)^{|\mu|}}= & \sum_{\substack{\text { nonattacking fillings } \\
\sigma \text { of } \mu^{\prime}}} X^{\sigma} q^{\operatorname{maj}\left(\sigma, \mu^{\prime}\right)+k \operatorname{coinv}\left(\sigma, \mu^{\prime}\right)} \\
& \times \prod_{\substack{u \in \mu^{\prime} \\
\sigma(u)=\sigma(\operatorname{South}(u))}}[\operatorname{leg}(u)+k \cdot \operatorname{arm}(u)+k+1]_{q} \prod_{\substack{u \in \mu^{\prime} \\
\sigma(u) \neq \sigma(\operatorname{South}(u))}}[k]_{q},
\end{aligned}
$$

hence the $q$-positivity is obvious in the monomial expansion.

\section{Computation}

From now on, we use the notation $A \amalg B$ to denote a shuffle product of two sequences of numbers $A=\left(a_{1}, \ldots, a_{k}\right)$ and $B=\left(b_{1}, \ldots, b_{r}\right)$, and $A \| B$ to denote the concatenation of two sequences $A$ and $B$. Also, given $\mu,|\mu|=n$, we identify a permutation $w \in S_{n}$ with the standard filling of $\mu$ whose reading word is $w$.

3.1. Computation of $\left\langle J_{\mu}(X ; q, t), s_{\left(k+1,1^{n-k-1}\right)}\right\rangle$. By Theorem 2.3, for $\lambda=(k+$ $\left.1,1^{n-k-1}\right)$, we know that $K_{n}^{*}(\alpha, \lambda)=\chi(\alpha=\lambda)$, and we can easily get that $w \in S_{n}$ satisfying $\operatorname{Des}^{\prime}(w)=\lambda$ is of the form

$$
w \in \mathcal{F}:=\{[(1,2, \ldots, k) ш(n, n-1, \ldots, k+2)] \| k+1\} .
$$

Before we start with simple cases, define an operator $\phi$ by

$$
\phi\left(\left(q^{a} t ;-\right)_{n}\right)=q^{n}\left(q^{a-1} t ;-\right)_{n} .
$$

Proposition 3.1.

$$
\left\langle J_{(n)}, s_{\left(k+1,1^{n-k-1}\right)}\right\rangle=\left(q^{-(n-k-1)} t ; q\right)_{n} \cdot q^{\left(\frac{n-k}{2}\right)}\left[\begin{array}{c}
n-1 \\
k
\end{array}\right]_{q},
$$

where $(a ; q)_{n}=(1-a)(1-a q) \cdots\left(1-a q^{n-1}\right)$.

Proof. We calculate $D_{(w,(n))}(q, t)$ for $w$ 's in (3.1) by induction. By considering $w$ 's in $\mathcal{F}$ as fillings of $\mu=(n)$, we can divide $\mathcal{F}$ into two subsets according to the element in the first cell of $\mu$ : (i) when 1 is in the first cell and (ii) when $n$ is in the first cell. We let

$$
\begin{aligned}
& \mathcal{F}_{1}=\{1\|[(2, \ldots, k) \amalg(n, n-1, \ldots, k+2)]\| k+1\}, \\
& \mathcal{F}_{2}=\{n\|[(1, \ldots, k) \amalg(n-1, \ldots, k+2)]\| k+1\} .
\end{aligned}
$$


After fixing the first element of $\mu$, we can use the induction hypothesis. In $\mathcal{F}_{1}$ case, if we ignore the first 1 in the fillings, then the rest (say $w^{\prime}$ ) forms the set of fillings for $\left\langle J_{(n-1)}, s_{\left(k, 1^{n-k-1}\right)}\right\rangle$, i.e.,

$$
w^{\prime} \in \mathcal{F}_{1}^{\prime}=\{[(2, \ldots, k) \amalg(n, n-1, \ldots, k+2)] \| k+1\} .
$$

By the induction hypothesis, we know that

$$
\begin{aligned}
\sum_{w^{\prime} \in \mathcal{F}_{1}^{\prime}} D_{\left(w^{\prime},(n-1)\right)}(q, t) & =\left\langle J_{(n-1)}, s_{\left(k, 1^{n-k-1}\right)}\right\rangle \\
& =\left(q^{-(n-k-1)} t ; q\right)_{n-1} \cdot q^{\left(\begin{array}{c}
n-k \\
2
\end{array}\right)}\left[\begin{array}{c}
n-2 \\
k-1
\end{array}\right]_{q} .
\end{aligned}
$$

If we put 1 in front of the the fillings in $\mathcal{F}_{1}^{\prime}$, then it would not change the factors in $D_{\left(w^{\prime},(n-1)\right)}(q, t)$ and the cell with 1 itself contributes $\left(1-q^{n-1} t\right)$ to $D_{(w,(n))}(q, t)$.

In the second case, if we let $\mathcal{F}_{2}^{\prime}=\{[(1, \ldots, k) \amalg(n-1, \ldots, k+2)] \| k+1\}$, then $\mathcal{F}_{2}^{\prime}$ is the set of fillings for $\left\langle J_{(n-1)}, s_{\left(k+1,1^{n-k-2}\right)}\right\rangle$ and again by the induction hypothesis, we have

$$
\left\langle J_{(n-1)}, s_{\left(k+1,1^{n-k-2}\right)}\right\rangle=\left(q^{-(n-2-k)} t ; q\right)_{n-1} \cdot q^{\left(\left(_{2}^{n-k-1}\right)\right.}\left[\begin{array}{c}
n-2 \\
k
\end{array}\right]_{q} .
$$

If we attach the cell with $n$ in front of the fillings in $\mathcal{F}_{2}^{\prime}$, then $n$ does not create any inversion or coinversion triples since $n$ is the largest number, but every entry to the right of $n$ gains one inversion created with $n$. Thus, the contribution to $D_{(w,(n))}(q, t)$ in this case would be

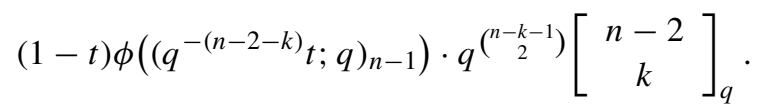

Hence, we get

$$
\begin{aligned}
\left\langle J_{(n)}, s_{\left(k+1,1^{n-k-1}\right)}\right\rangle= & \left(1-q^{n-1} t\right) \cdot\left(\left(q^{-(n-k-1)} t ; q\right)_{n-1} \cdot q^{\left(\frac{n-k}{2}\right)}\left[\begin{array}{c}
n-2 \\
k-1
\end{array}\right]_{q}\right) \\
& +(1-t) \phi\left(\left(q^{-(n-2-k)} t ; q\right)_{n-1}\right) \cdot q^{\left(\frac{n-k-1}{2}\right)}\left[\begin{array}{c}
n-2 \\
k
\end{array}\right]_{q} \\
= & \left(q^{-(n-k-1)} t ; q\right)_{n} \cdot q^{\left(\frac{n-k}{2}\right)}\left[\begin{array}{c}
n-1 \\
k
\end{array}\right]_{q} .
\end{aligned}
$$

Note that $\phi\left(\left(q^{-(n-2-k)} t ; q\right)_{n-1}\right)=q^{n-1}\left(q^{-(n-k-1)} t ; q\right)_{n-1}$.

We apply a similar idea of using induction to find the Schur coefficient of $J_{\mu}$ when the Schur function is indexed by hook shapes. 
THEOREM 3.2. For any $\mu \vdash n$,

$$
\begin{aligned}
\left\langle J_{\mu}, s_{\left(k+1,1^{n-k-1}\right)}\right\rangle= & {\left[\prod_{i=1}^{l} q^{(i-1)\left(\mu_{i}^{\prime}-1\right)}\left(q^{-(i-1)} t ; t\right)_{\mu_{i}^{\prime}-1}\left(1-q^{k-i+1} t^{\mu_{i}^{\prime}}\right)\right] } \\
& \left.\times\left(q^{-\left(\mu_{1}-k\right)-1} t ; q\right)_{m} \cdot q^{\left({ }^{\mu_{1}-k}\right)}{ }_{2}\right)\left[\begin{array}{c}
\mu_{1}-1 \\
k
\end{array}\right]_{q},
\end{aligned}
$$

where $l+m=\mu_{1}$ and $l$ is the number of $i$ 's with $\mu_{i}^{\prime}>1$ and $m$ is the number of $i$ 's with $\mu_{i}^{\prime}=1$.

PROOF. Recall that we are only considering the fillings of the form

$$
w \in \mathcal{F}=\{[(1,2, \ldots, k) ш(n, n-1, \ldots, k+2)] \| k+1\}
$$

which satisfy $\operatorname{Des}^{\prime}(w)=\lambda$ for $\lambda=\left(k+1,1^{n-k-1}\right)$. Also, note that due to the primary condition for the fillings of $\mu$, the entry 1 should be in the first row, and since the numbers in the set $(1,2, \ldots, k)$ should be in that relative order in the fillings of $\mu$, all the numbers in the set $(1,2, \ldots, k)$ come in the first row. Hence, we fill the cells in $\mu$ with numbers from $n$ in decreasing order, from left to right, starting from the top row (i.e., in reading order) up to the second row. This part of the filling will be fixed. Then we only have to consider the fillings of the first row which are of the form

$$
\left\{\left[(1,2, \ldots, k) ш\left(\mu_{1}, \mu_{1}-1, \ldots, k+3, k+2\right)\right] \| k+1\right\} .
$$

Having this observation, we can divide the set of fillings $\mathcal{F}$ in two subsets: when 1 is in the $(1,1)$ cell, and when $\mu_{1}$ (which is the largest possible entry in the first row) comes in the $(1,1)$ cell. If we fix the entry in the $(1,1)$ cell, then consequently we get to fix all the elements in the first column, so we can use the induction hypothesis for the parts to the right of the first column. If we remove the first column of $\mu$ and let $\tilde{\mu}=\left(\mu_{1}-1, \mu_{2}-1, \ldots\right)$, then by only considering the relative order of fillings of $\tilde{\mu}$, we get $\left\langle J_{\tilde{\mu}}, s_{\left(k, 1^{n-\mu_{1}-k}\right)}\right\rangle$ which is

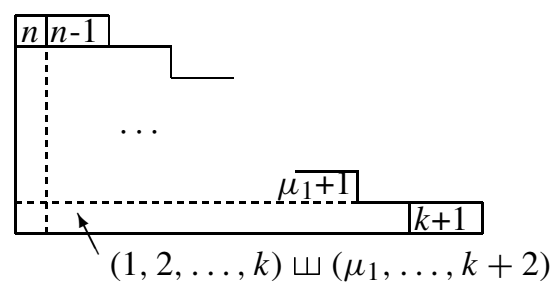

FIGURE 2. Fillings of $\mu$ 


$$
\begin{aligned}
\left\langle J_{\tilde{\mu}}, s_{\left(k, 1^{\left.n-\mu_{1}-k\right)}\right\rangle}\right\rangle & =\left[\prod_{i=1}^{l-1} q^{(i-1)\left(\mu_{i+1}^{\prime}-1\right)}\left(q^{-(i-1)} t ; t\right)_{\mu_{i+1}^{\prime}-1}\left(1-q^{k-i} t^{\left.\mu_{i+1}^{\prime}\right)}\right]\right. \\
& \times\left(q^{-\left(\mu_{1}-k\right)-1} t ; q\right)_{m} q^{\left({ }^{\mu_{1}-k}\right)}\left[\begin{array}{c}
\mu_{1}-2 \\
k-1
\end{array}\right]_{q} \\
= & {\left[\prod_{i=2}^{l} q^{(i-2)\left(\mu_{i}^{\prime}-1\right)}\left(q^{-(i-2)} t ; t\right)_{\mu_{i}^{\prime}-1}\left(1-q^{k-i+1} t^{\mu_{i}^{\prime}}\right)\right] } \\
& \times\left(q^{-\left(\mu_{1}-k\right)-1} t ; q\right)_{m} q^{\left({ }^{\mu_{1}-k}\right)}\left[\begin{array}{c}
\mu_{1}-2 \\
k-1
\end{array}\right]_{q} .
\end{aligned}
$$

If we attach the first column containing 1 in the $(1,1)$ cell, then the first column itself gives

$$
D_{\left(\left.w\right|_{1} \mu_{1}^{\prime}, 1^{\mu_{1}^{\prime}}\right.}(q, t)=(t ; t)_{\mu_{1}^{\prime}-1}\left(1-q^{\mu_{1}-1} t^{\mu_{1}^{\prime}}\right)
$$

and since the elements in the first column are the largest in each row, the entries above the first row to the right of the first column would gain one inversion. Thus, this subset of fillings contributes the following to $D_{(w, \mu)}(q, t)$ :

$$
\begin{gathered}
\begin{aligned}
(t ; t)_{\mu_{1}^{\prime}-1}\left(1-q^{\mu_{1}-1} t_{1}^{\mu^{\prime}}\right)\left[\prod_{i=2}^{l} q^{(i-2)\left(\mu_{i}^{\prime}-1\right)} \phi\left(\left(q^{-(i-2)} t ; t\right)_{\mu_{i}^{\prime}-1}\right)\left(1-q^{k-i+1} t^{\mu_{i}^{\prime}}\right)\right] \\
\times\left(q^{-\left(\mu_{1}-k\right)-1} t ; q\right)_{m} q^{\left({ }^{\mu_{1}-k}\right)}\left[\begin{array}{c}
\mu_{1}-2 \\
k-1
\end{array}\right]_{q}
\end{aligned} \\
\begin{array}{r}
(3.5)=(t ; t)_{\mu_{1}^{\prime}-1}\left(1-q^{\mu_{1}-1} t^{\mu_{1}^{\prime}}\right)\left[\prod_{i=2}^{l} q^{(i-1)\left(\mu_{i}^{\prime}-1\right)}\left(q^{-(i-1)} t ; t\right)_{\mu_{i}^{\prime}-1}\left(1-q^{k-i+1} t^{\mu_{i}^{\prime}}\right)\right] \\
\times\left(q^{-\left(\mu_{1}-k\right)-1} t ; q\right)_{m} q_{2}^{\left(\mu_{1}-k\right)}\left[\begin{array}{c}
\mu_{1}-2 \\
k-1
\end{array}\right]_{q} .
\end{array}
\end{gathered}
$$

Similarly, if we consider the subset of fillings with $\mu_{1}$ in the $(1,1)$ cell, the fillings of $\tilde{\mu}_{1}$ part would give $\left\langle J_{\tilde{\mu}}, s_{\left(k+1,1^{n-\mu_{1}-k-1}\right)}\right\rangle$ and the first column itself gives $\left(1-t^{\mu_{1}^{\prime}}\right)(t ; t)_{\mu_{1}^{\prime}-1}$. 
Attaching the first column to $\tilde{\mu}$ would give

$$
\begin{array}{r}
\left(1-t^{\mu_{1}^{\prime}}\right)(t ; t)_{\mu_{1}^{\prime}-1}\left[\prod_{i=2}^{l} q^{(i-2)\left(\mu_{i}^{\prime}-1\right)} \phi\left(\left(q^{-(i-2)} t ; t\right)_{\mu_{i}^{\prime}-1}\right)\left(q-q^{k-i+2} t^{\mu_{i}^{\prime}}\right)\right] \\
\left.\times \phi\left(\left(q^{-\left(\mu_{1}-k\right)} t ; q\right)_{m}\right) \cdot q^{\left(\mu_{1}-k-1\right.}\right)\left[\begin{array}{c}
\mu_{1}-2 \\
k
\end{array}\right]_{q} \\
=\left(1-t^{\mu_{1}^{\prime}}\right)(t ; t)_{\mu_{1}^{\prime}-1}\left[\prod_{i=2}^{l} q^{(i-1)\left(\mu_{i}^{\prime}-1\right)}\left(q^{-(i-1)} t ; t\right)_{\mu_{i}^{\prime}-1}\left(1-q^{k-i+1} t^{\mu_{i}^{\prime}}\right)\right] \\
\times\left(q^{-\left(\mu_{1}-k\right)-1} t ; q\right)_{m} \cdot q^{k+\left({ }^{\mu_{1}-k}\right)}\left[\begin{array}{c}
\mu_{1}-2 \\
k
\end{array}\right] .
\end{array}
$$

Adding (3.5) and (3.6) gives $\left\langle J_{\mu}, s_{\left(k+1,1^{n-k-1}\right)}\right\rangle$.

3.2. Two row case. We make the following observation which will be used to prove several Schur coefficient formulas of $J_{(b, a)}(X ; q, t)$. Let $n=a+b$.

LEMMA 3.3. Let $\mu=(b, a)$. Consider the following set of permutations (identified with the standard fillings of $\mu$ whose reading words are corresponding permutations) for $r, s \leq a$

$$
\mathcal{F}=\{[(n, n-1, \ldots, n-(a-s-1)) \amalg(r+1, \ldots, r+s)] \|
$$

$[(1,2, \ldots, r) ш(r+s+1, r+s+2, \ldots, a+s)] \|(a+s+1, a+s+2, \ldots, n-a+s)\}$.

If $r<s$, then for any $w \in \mathcal{F}$,

$$
D_{(w, \mu)}(q, t)=0 .
$$

Proof. Note that the elements of $\mathcal{F}$ fill the second row (top row) of $\mu$ with $(n, n-$ $1, \ldots, n-(a-s-1)) ш(r+1, \ldots, r+s)$ and the first row (bottom row) with $(1,2, \ldots, r) ш$ $(r+s+1, r+s+2, \ldots, a+s)$ from the left, and $(a+s+1, a+s+2, \ldots, n-a+s)$ will

be fixed in the tail part of the first row. We use the notation $[\alpha: \beta]$ to denote the domino $\bar{\beta}$ which is a column of $\mu$. For any fixed $s, s>r$, we use the induction on $r$.

Let $r=1$. If $r=1$ is below one of the numbers $(n, n-1, \ldots, n-(a-s-1))$, i.e., if we have a domino of the form $[n-\alpha: 1]$ for $0 \leq \alpha \leq a-s-1$, then there also exists a domino $[2: s+\beta]$ for $2 \leq \beta \leq a$. If the domino [ $n-\alpha: 1]$ comes to the right of $[2: s+\beta]$, then since 2 is the smallest number in any triples containing 2, if we let $c$ be the cell containing 2, then $\operatorname{inv}_{c}(w, \mu)=\operatorname{coinv}_{c}(w, \mu)=0$. Thus $c$ contributes $(t-t)=0$ to $D_{(w, \mu)}(q, t)$ which

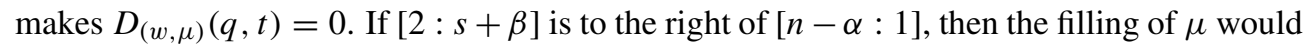
have three dominos in the following order

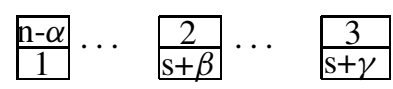


where $\gamma>\beta$. The entries to the left of $[3: s+\gamma]$ are all greater than 3 except 1 and 2 , so if we say the cell containing 3 is $c$, then $\operatorname{inv}_{c}(w, \mu)=\operatorname{coinv}_{c}(w, \mu)=1$. Hence the cell $c$ contributes $(q t-q t)=0$ to $D_{(w, \mu)}(q, t)$ which also makes $D_{(w, \mu)}(q, t)$ zero. Now consider the case when $r=1$ is below one of the numbers in $(r+1, r+2, \ldots, r+s)$. If it is below $r+1$, i.e., if there is a domino $[r+1: 1]$, then there would also be a domino $[r+2: r+s+\alpha]$ where $1 \leq \alpha \leq a-r$ to the right of the domino $[r+1: 1]$. Since $r+2$ is the smallest number among the numbers consisting triples with it, the cell filled with $r+2$ contributes $(t-t)=0$ to $D_{(w, \mu)}(q, t)$. If 1 is below $r+\beta$ for $\beta>1$, then $r+1$ would be above a number of the form $r+s+\beta$ which is larger than $r+1$. Also, $r+1$ is the smallest among the numbers consisting triples with it, so it contributes $(t-t)=0$ to $D_{(w, \mu)}(q, t)$. This proves the case when $r=1$.

Now we assume that the lemma is true for $1 \leq r \leq s-2$. We can divide $\mathcal{F}$ into four subsets with respect to the form of the first column domino; (i) fillings starting with [ $n: 1]$, (ii) fillings starting with $[r+1: r+s+1]$, (iii) fillings starting with $[r+1: 1]$ and (iv) fillings starting with $[n: r+s+1]$.

(i) If the first column of $\mu$ is $[n: 1]$, then the rest part of $\mu$ would be filled with ( $n-$ $1, \ldots, n-(a-s-1)) \amalg(r+1, \ldots, r+s)$ in the second row to the right of $n$, and $(2, \ldots, r) ш$ $(r+s+1, r+s+2, \ldots, a+s)$ in the first row to the right of 1 . If we let

$$
\begin{gathered}
\tilde{\mathcal{F}}=\{[(n-1, \ldots, n-(a-s-1)) ш(r+1, \ldots, r+s)] \| \\
[(2, \ldots, r) \amalg(r+s+1, r+s+2, \ldots, a+s)] \|(a+s+1, a+s+2, \ldots, n-a+s)\},
\end{gathered}
$$

and $\tilde{\mu}=(b-1, a-1)$, then by the induction hypothesis, we have

$$
D_{(\tilde{w}, \tilde{\mu})}(q, t)=0
$$

for any $\tilde{w} \in \tilde{\mathcal{F}}$. In fact, as we saw in $r=1$ case, for each $\tilde{w} \in \tilde{\mathcal{F}}$, we can find a domino $[r+\alpha: r+s+\beta]$ which contributes 0 to $D_{(\tilde{w}, \tilde{\mu})}(q, t)$ due to the fact that the cell containing $r+\alpha$ has the same coinversion and inversion statistics. Note that since the largest possible coinv value is $s-2$ in this case and $s-1$ for inv, there exists a domino $[r+\tilde{\alpha}: r+s+\tilde{\beta}]$ to the right of $[r+\alpha: r+s+\beta]$ for which the cell with $r+\tilde{\alpha}$ has one more inversion statistic than $r+\alpha$, having the same coinversion statistic. If we attach the domino [n:1] in front of $\tilde{\mu}$ filed with $\tilde{w} \in \tilde{\mathcal{F}}$, then every entry in the set $(r+1, \ldots, r+s)$ gains one coinv, and so the cell filled with $r+\tilde{\alpha}$ gets to have the same number of coinversions and inversions. So this gives 0 to $D_{(w, \mu)}(q, t)$. In case (ii), the cell containing $r+1$ contributes $(t-t)=0$ to $D_{(w, \mu)}(q, t)$, since $r+1$ is the smallest among the entries consisting triples with it, so it does not create any coinversion or inversion statistics.

In cases (iii) and (iv), we cannot use the induction hypothesis. Say we make a filling of $\mu$ by only using type (iii) or type (iv) dominos starting from the first column. Note that if we put type (i) or type (ii) domino in this process, we can apply the induction hypothesis there and derive $D_{(w, \mu)}=0$. Type (iii) dominos pair the numbers in the set $(r+1, r+2, \ldots, r+s)$ which has $s$ elements and the set $(1,2, \ldots, r)$ which has $r=s-1$ elements. Also, type (ii) 
domino pairs the numbers in the set $(n, n-1, \ldots, n-a+s+1)$ with $a-s$ elements and the numbers in the set $(r+s+1, \ldots, a+s)$ with $a-r=a-s+1$ elements. Due to the discrepancy of the number of elements in two sets, there should be a domino of the form $[r+\alpha: r+s+\beta]$. If there are only type (iii) or type (iv) dominos to the left of this one, then the cell containing $r+\alpha$ has no coinversions or inversions, and so it contributes 0 to $D_{(w, \mu)}(q, t)$. Thus we prove the lemma.

We start by considering the coefficients of the Schur functions indexed by two-row shapes.

Proposition 3.4. For $0 \leq k \leq \frac{b-a}{2}$,

$$
\begin{aligned}
& \left\langle J_{(b, a)}(X ; q, t), s_{(b-k, a+k)}\right\rangle \\
& \quad=(t ; q)_{a}\left(q^{b-a} t^{2} ; q\right)_{a}\left(q^{-1} t ; q\right)_{k}(t ; q)_{b-a-k} q^{k}\left[\begin{array}{c}
b-a+1 \\
k
\end{array}\right]_{q} .
\end{aligned}
$$

Proof. There are two special flat rim-hook tableaux of shape $\lambda=(b-k, a+k)$ :
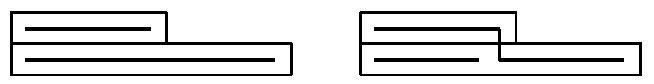

The composition $\alpha_{l}$ corresponding to the left rim-hook tableau is $(b-k, a+k)$ and the set of $w \in S_{n}$ satisfying $\operatorname{Des}^{\prime}(w)=\alpha_{l}$ is

$$
\mathcal{F}_{l}=\{(1,2, \ldots, b-k) ш(b-k+1, b-k+2, \ldots, n) \mid b-k+1 \text { precedes } b-k\} .
$$

Similarly, the right rim-hook tableau gives $\alpha_{r}=(a+k-1, b-k+1)$ and the set of $w \in S_{n}$ satisfying $\operatorname{Des}^{\prime}(w)=\alpha_{r}$ is

$$
\mathcal{F}_{r}=\{(1,2, \ldots, a+k-1) \amalg(a+k, a+k+1, \ldots, n) \mid a+k \text { precedes } a+k-1\} .
$$

Note that due to the primary condition, the elements in the set $(1,2, \ldots)$ should be in the first row, so the filling in the second row would be fixed, for any $w \in \mathcal{F}_{l} \cup \mathcal{F}_{r}$, by the elements if the second set of the shuffle product. Also, since the entries in the second row are increasing from the left, notice that if we have a triple

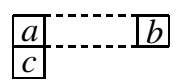

with $a<b<c$, $a$ gets to have no coinversions or inversions, so the cell containing $a$ contributes 0 to $D_{(w, \mu)}(q, t)$. This implies that we cannot have a domino of the form $[\alpha: \beta]$ with $\beta>\alpha$, hence the first $a$ entries of the first row would be also fixed by $(1,2, \ldots, a)$. So the entries in the part with column height 2 are fixed and they contribute $(t ; q)_{a}\left(q^{b-a} t^{2} ; q\right)_{a}$ to $D_{(w, \mu)}(q, t)$ for $w \in \mathcal{F}_{l} \cup \mathcal{F}_{r}$. Having the fillings of the two-rowed part fixed, we only have to consider the factors from the tail part of size $b-a$.

Let

$$
\tilde{F}_{l}=\{(a+1, a+2, \ldots, b-k) \amalg(a+b-k+1, a+b-k+2, \ldots, a+b)\},
$$




$$
\tilde{F}_{r}=\{(a+1, a+2, \ldots, a+k-1) \amalg(2 a+k, 2 a+k+1, \ldots, a+b)\} .
$$

By using induction, it is not too hard to show that

$$
\begin{aligned}
\sum_{\tilde{w} \in \tilde{F}_{l}} D_{(\tilde{w},(b-a))}(q, t) & =(t ; q)_{k}(t ; q)_{b-a-k}\left[\begin{array}{c}
b-a \\
k
\end{array}\right]_{q}, \\
\sum_{\tilde{w} \in \tilde{F}_{r}} D_{(\tilde{w},(b-a))}(q, t) & =(t ; q)_{k-1}(t ; q)_{b-a-k+1}\left[\begin{array}{c}
b-a \\
k-1
\end{array}\right]_{q} .
\end{aligned}
$$

Since $K_{n}^{*}\left(\alpha_{l}, \lambda\right)=+1$ and $K_{n}^{*}\left(\alpha_{r}, \lambda\right)=-1$, we have

$$
\begin{aligned}
\left\langle J_{(b, a)}\right. & \left.(X ; q, t), s_{(b-k, a+k)}\right\rangle \\
= & (t ; q)_{a}\left(q^{b-a} t^{2} ; q\right)_{a}\left(\sum_{\tilde{w} \in \tilde{F}_{l}} D_{(\tilde{w},(b-a))}(q, t)-\sum_{\tilde{w} \in \tilde{F}_{r}} D_{(\tilde{w},(b-a))}(q, t)\right) \\
= & (t ; q)_{a}\left(q^{b-a} t^{2} ; q\right)_{a}\left((t ; q)_{k}(t ; q)_{b-a-k}\left[\begin{array}{c}
b-a \\
k
\end{array}\right]_{q}\right. \\
& \left.-(t ; q)_{k-1}(t ; q)_{b-a-k+1}\left[\begin{array}{c}
b-a \\
k-1
\end{array}\right]_{q}\right) \\
= & (t ; q)_{a}\left(q^{b-a} t^{2} ; q\right)_{a}\left(q^{-1} t ; q\right)_{k}(t ; q)_{b-a-k} q^{k}\left[\begin{array}{c}
b-a+1 \\
k
\end{array}\right]_{q} .
\end{aligned}
$$

Now we consider the case when the Schur functions are indexed by $\lambda=\left(r, s, 1^{k}\right)$ shapes. Note that there are two special flat rim-hook tableaux of shape $\lambda$ as in Figure 3.
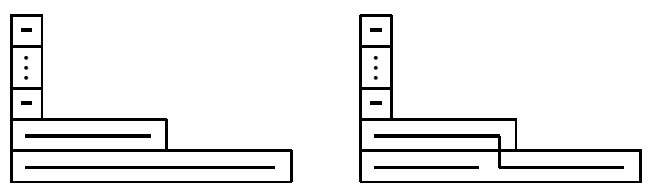

FIGURE 3. Two special flat rim-hook tableaux of shape $\lambda=\left(r, s, 1^{k}\right)$

The tableau in the left hand side has $K_{n}^{*}(\alpha, \lambda)=+1$ and the right hand side one has $K_{n}^{*}(\alpha, \lambda)=-1$.

PROPOSITION 3.5 .

$$
\left\langle J_{(b, a)}(X, q, t), s_{\left(b, a-k, 1^{k}\right)}\right\rangle=\left(q^{-k} t ; q\right)_{a}\left(q^{b-a} t^{2} ; q\right)_{a}(t ; q)_{b-a} \cdot q^{\left(\begin{array}{c}
k+1 \\
2
\end{array}\right)}\left[\begin{array}{c}
a-1 \\
k
\end{array}\right]_{q} .
$$


Proof. Let $n=a+b$. Let $\mathcal{F}_{1}(\lambda)$ be the set of fillings $w$ for which $\operatorname{Des}^{\prime}(w)$ corresponds to the first special rim-hook tableaux of Figure 3. That is,

$$
\begin{aligned}
\mathcal{F}_{1}(\lambda)=\{(1,2, \ldots, b) & (b+1, \ldots, n-k) \amalg(n, n-1, \ldots, n-k+1) \\
& \text { such that } b+1 \text { precedes } b, \text { and } n-k+1 \text { precedes } n-k\} .
\end{aligned}
$$

Similarly, we define the set of the fillings, $\mathcal{F}_{2}(\lambda)$, corresponding to the second special rimhook tableau of Figure 3.

$$
\mathcal{F}_{2}(\lambda)=\{(1,2, \ldots, a-k-1) \amalg(a-k, \ldots, n-k) \amalg(n, n-1, \ldots, n-k+1)
$$

such that $a-k$ precedes $a-k-1$, and $n-k+1$ precedes $n-k\}$.

We first consider $w \in \mathcal{F}_{2}(\lambda)$. Due to the primary condition, the elements in the set $(1,2, \ldots, a-k-1)$ should be in the first row, and so the numbers in the second and the third set which are larger than the numbers in the set $(1,2, \ldots, a-k-1)$ get to fill the second row. Then in the language of Lemma 3.3, this is the case when $r \leq a-k-1$ and $s \geq a-k$, and so $r<s$. Hence, by Lemma 3.3, $D_{(w,(b, a))}(q, t)=0$ for any $w \in \mathcal{F}_{2}(\lambda)$. So we only have to compute $D_{(w,(b, a))}(q, t)$ for $w \in \mathcal{F}_{1}(\lambda)$, and the primary condition fixes the entries in the first row by $(1,2, \ldots, b)$ in this case. Then in the second row, we have

$$
\begin{array}{r}
\tilde{\mathcal{F}}_{1}(\lambda)=\{(a+b, a+b-1, \ldots, a+b-k+1) \\
\text { such that } a+b-k+1, b+2, \ldots, a+b-k) \\
\text { precedes } a+b-k\} .
\end{array}
$$

Notice that $\sum_{w \in \tilde{\mathcal{F}}_{1}(\lambda)} D_{(w, \mu)}(q, t)$ is nothing but $\left\langle J_{(a)}, s_{\left(a-k, 1^{k}\right)}\right\rangle$ and by Proposition 3.1, we know that

$$
\left\langle J_{(a)}, s_{\left(a-k, 1^{k}\right)}\right\rangle=\left(q^{-k} t ; q\right)_{a} \cdot q^{\left(\begin{array}{c}
k+1 \\
2
\end{array}\right)}\left[\begin{array}{c}
a-1 \\
k
\end{array}\right]_{q} .
$$

Combining the factors from the first row, we get

$$
\left\langle J_{(b, a)}(X, q, t), s_{\left(b, a-k, 1^{k}\right)}\right\rangle=\left(q^{-k} t ; q\right)_{a}\left(q^{b-a} t^{2} ; q\right)_{a}(t ; q)_{b-a} \cdot q^{\left(\begin{array}{c}
k+1 \\
2
\end{array}\right)}\left[\begin{array}{c}
a-1 \\
k
\end{array}\right]_{q} .
$$

Proposition 3.6. For $0 \leq k \leq a-1$,

$$
\begin{aligned}
\left\langle J_{(b, a)}(X ; q, t), s_{\left(b-1, a-k, 1^{k+1}\right)}\right\rangle= & \left(t ; q^{-1}\right)_{k+1}(t ; q)_{a-k-1}\left(q^{b-a} t^{2} ; q\right)_{a-1}\left(q^{-1} t ; q\right)_{b-a} \\
& \times\left(1-q^{b-k-2} t^{2}\right) q^{\left({ }^{k+1}\right)+1}[b-a+k]_{q}\left[\begin{array}{c}
a \\
k+1
\end{array}\right]_{q} .
\end{aligned}
$$

PROOF. We also have two special flat rim-hook tableaux of shape $\lambda=(b-1, a-$ $k, 1^{k+1}$ ) as in Figure 3. Considering the primary condition for the fillings, we get the set of 
fillings corresponding to the special rim-hook tableaux from the left $\mathcal{F}_{l}=\{(1,2, \ldots, b-1) ш(b, b+1, \ldots, n-k-1) ш(n, n-1, \ldots, n-k)$ such that $b$ precedes $b-1$ and $n-k$ precedes $n-k-1\}$, $\mathcal{F}_{r}=\{(1,2, \ldots, a-k-1) \amalg(a-k, a-k+1, \ldots, n-k-1) \amalg(n, n-1, \ldots, n-k)$ such that $a-k$ precedes $a-k-1$ and $n-k$ precedes $n-k-1\}$.

Note that due to Lemma 3.3, we only have to consider the following subsets of $\mathcal{F}_{l}$ and $\mathcal{F}_{r}$ :

$$
\begin{aligned}
& \mathcal{F}_{l}^{1}=\{[(n, n-1, \ldots, n-k) \amalg(b, b+1, \ldots, n-k-2)] \\
& \|(1,2, \ldots, a)\|[(a+1, \ldots, b-1) \amalg n-k-1]\}, \\
& \mathcal{F}_{l}^{2}=\{[(n, n-1, \ldots, n-k) \amalg(b, b+1, \ldots, n-k-2)] \\
& \|[(1,2, \ldots, a-1) \amalg n-k-1]\|(a, a+1, \ldots, b-1)\}, \\
& \tilde{\mathcal{F}}_{r}=\{[(n, n-1, \ldots, n-k) \amalg(a-k, a-k+1, \ldots, 2 a-2 k-2)] \\
& \|[(1,2, \ldots, a-k-1) \amalg(2 a-2 k-1, \ldots, 2 a-k-1)]\|(2 a-k, \ldots, n-k-1)\} .
\end{aligned}
$$

The fillings in $\mathcal{F}_{l} \cup \mathcal{F}_{r}$ which are not contained in the above three subsets are $w$ 's satisfying $D_{(w, \mu)}(q, t)=0$. The computation of $D_{(w, \mu)}(q, t)$ is a routine.

$$
\sum_{w \in \mathcal{F}_{l}^{1}} D_{(w, \mu)}(q, t)=(1-t)\left(t ; q^{-1}\right)_{k+1}(t ; q)_{a-k-1}\left(q^{b-a} t^{2} ; q\right)_{a}(t ; q)_{b-a-1}
$$

$$
\times q^{\left({ }_{2}\right)}[b-a]_{q}\left[\begin{array}{c}
a \\
k+1
\end{array}\right]_{q},
$$

$$
\sum_{w \in \mathcal{F}_{l}^{2}} D_{(w, \mu)}(q, t)=\left(t ; q^{-1}\right)_{k+1}(t ; q)_{a-k-1}\left(q^{b-a} t^{2} ; q\right)_{a-1}\left(q^{-1} t ; q\right)_{b-a}
$$

$$
\times\left(1-q^{a-k-1} t^{2}\right) q^{\left(\begin{array}{c}
k+1 \\
2
\end{array}\right)+b-a}[k+1]_{q}\left[\begin{array}{c}
a \\
k+1
\end{array}\right]_{q}
$$

$$
\sum_{w \in \tilde{\mathcal{F}}_{r}} D_{(w, \mu)}(q, t)=\left(t ; q^{-1}\right)_{k+1}(t ; q)_{a-k-1}\left(q^{b-a} t^{2} ; q\right)_{a}(t ; q)_{b-a} q^{\left(\begin{array}{c}
k+1 \\
2
\end{array}\right)}\left[\begin{array}{c}
a \\
k+1
\end{array}\right]_{q} .
$$

Considering the values of $K_{n}^{*}(\alpha, \lambda)$, we get

$$
\begin{aligned}
& \left\langle J_{(b, a)}(X ; q, t), s_{\left(b-1, a-k, 1^{k+1}\right)}\right\rangle=(3.8)+(3.9)-(3.10) \\
& =\left(t ; q^{-1}\right)_{k+1}(t ; q)_{a-k-1}\left(q^{b-a} t^{2} ; q\right)_{a-1}\left(q^{-1} t ; q\right)_{b-a} \\
& \quad \times\left(1-q^{b-k-2} t^{2}\right) q^{\left(\begin{array}{c}
k+1 \\
2
\end{array}\right)+1}[b-a+k]_{q}\left[\begin{array}{c}
a \\
k+1
\end{array}\right]_{q} .
\end{aligned}
$$


PROPOSITION 3.7. For $0 \leq k \leq \frac{b-a-1}{2}$,

$$
\begin{gathered}
\left\langle J_{(b, a)}(X, q, t), s_{(b-k-1, a+k, 1)}\right\rangle=(1-t)(t ; q)_{a-1}(t ; q)_{k-1}\left(q^{b-a} t^{2} ; q\right)_{a-1}\left(q^{-1} t ; q\right)_{b-a-k} \\
\times q^{k+1}[a]_{q}\left[\begin{array}{c}
b-a \\
k
\end{array}\right]_{q} \frac{[b-a-2 k]_{q}}{[b-a-k+1]_{q}[k+1]_{q}} \\
\times\left\{\left(1-q^{b-a-k-1} t\right)\left(1-q^{b-1} t^{2}\right)[k]_{q}+q^{k-1}(q-t)\left(1-q^{b-k-2} t^{2}\right)[b-a-k+1]_{q}\right\} \\
+(t ; q)_{a}\left(q^{b-a} t^{2} ; q\right)_{a}(t ; q)_{k-1}(t ; q)_{b-a-k-1}(q-t)\left(q^{2}-t\right) q^{k-1} \frac{[k]_{q}[b-a-2 k]_{q}}{[b-a-k+1]_{q}}\left[\begin{array}{c}
b-a \\
k+1
\end{array}\right]_{q} .
\end{gathered}
$$

Proof. There are two special flat rim-hook tableaux of shape $(b-k-1, a+k, 1)$. Each gives the following set of fillings:
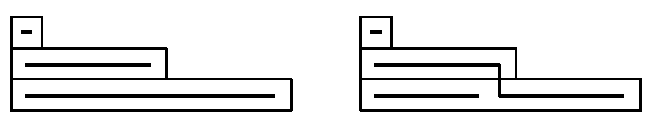

FIGURE 4. Two special flat rim-hook tableaux of shape $\lambda=(b-k-1, a+k, 1)$

$$
\begin{aligned}
& \mathcal{F}_{1}=\{(1,2, \ldots, b-k-1) \amalg(b-k, b-k+1, \ldots, n-1) \amalg n \\
&\text { such that } b-k \text { precedes } b-k-1 \text { and } n \text { precedes } n-1\}, \\
& \mathcal{F}_{2}=\{(1,2, \ldots, a+k-1) \amalg(a+k, a+k+1, \ldots, n-1) \amalg n \\
&\text { such that } a+k \text { precedes } a+k-1 \text { and } n \text { precedes } n-1\} .
\end{aligned}
$$

We consider the following three subsets of $\mathcal{F}_{1}$ :

$$
\begin{gathered}
\mathcal{F}_{1}^{1}=\{(b-k, b-k+1, \ldots, n-k-1)\|(1,2, \ldots, a)\| \\
{[(a+1, a+2, \ldots, b-k-1) \amalg(n-k, n-k+1, \ldots, n-1) \amalg n]} \\
\quad \text { such that } n \text { precedes } n-1\}, \\
\mathcal{F}_{1}^{2}=\{[(b-k, b-k+1, \ldots, n-k-2) \amalg(a+b)]\|(1,2, \ldots, a)\| \\
[(a+1, a+2, \ldots, b-k-1) \amalg(n-k-1, n-k, \ldots, n-1)]\}, \\
\mathcal{F}_{1}^{3}=\{[(b-k, b-k+1, \ldots, n-k-2) \amalg n]\|[(1,2, \ldots, a-1) \amalg(n-k-1)]\| \\
\quad[(a, a+1, \ldots, b-k-1) \amalg(n-k, n-k+1, \ldots, n-1)]\} .
\end{gathered}
$$


Note that the fillings in $\mathcal{F}_{1}$ which are not contained in any of $\mathcal{F}_{1}^{i}$ for $i=1,2,3$ make $D_{(w, \mu)}(q, t)$ zero by Lemma 3.3. By computation, we get

$$
\sum_{w \in \mathcal{F}_{1}^{1}} D_{(w, \mu)}(q, t)=(t ; q)_{a}\left(q^{b-a} t^{2} ; q\right)_{a}(t ; q)_{k}\left(q^{-1} t ; q\right)_{b-a-k} q[k]_{q}\left[\begin{array}{c}
b-a \\
k+1
\end{array}\right]_{q},
$$$$
\sum_{w \in \mathcal{F}_{1}^{2}} D_{(w, \mu)}(q, t)
$$

$$
=(1-t)(t ; q)_{a-1}\left(q^{b-a} t^{2} ; q\right)_{a}(t ; q)_{k+1}(t ; q)_{b-a-k-1}[a]_{q}\left[\begin{array}{c}
b-a \\
k+1
\end{array}\right]_{q},
$$

and

$$
\sum_{w \in \mathcal{F}_{1}^{3}} D_{(w, \mu)}(q, t)
$$$$
=(1-t)(t ; q)_{a-1}\left(1-q^{a+k-1} t^{2}\right)\left(q^{b-a} t^{2} ; q\right)_{a-1}\left(q^{-1} t ; q\right)_{b-a-k}(t ; q)_{k} q^{b-a-k}[a]_{q}\left[\begin{array}{c}
b-a \\
k
\end{array}\right]_{q} \text {. }
$$

Similarly, we divide $\mathcal{F}_{2}$ into three subsets :

$$
\begin{gathered}
\mathcal{F}_{2}^{1}=\{(a+k, a+k+1, \ldots, a+k+a-1)\|(1,2, \ldots, a)\| \\
{[(a+1, a+2, \ldots, a+k-1) \amalg(2 a+k, 2 a+k+1, \ldots, n-1) \amalg n]} \\
\text { such that } n \text { precedes } n-1\}, \\
\mathcal{F}_{2}^{2}=\{[(a+k, a+k+1, \ldots, 2 a+k-2) \amalg n]\|(1,2, \ldots, a)\| \\
[(a+1, a+2, \ldots, a+k-1) \amalg(2 a+k-1, \ldots, n-1)]\}, \\
\mathcal{F}_{2}^{3}=\{[(a+k, a+k+1, \ldots, 2 a+k-2) \amalg n]\|[(1,2, \ldots, a-1) \amalg(2 a+k-1)]\| \\
[(a, a+1, \ldots, a+k-1) \amalg(2 a+k, 2 a+k+1 \ldots, n-1)]\} .
\end{gathered}
$$

Again, the fillings in $\mathcal{F}_{2}$ which are not considered in the above subsets $\mathcal{F}_{2}^{i}$, for $i=1,2,3$, are those which make $D_{(w, \mu)}(q, t)$ zero by Lemma 3.3. By computation, we get

$$
\sum_{w \in \mathcal{F}_{2}^{1}} D_{(w, \mu)}(q, t)=(t ; q)_{a}\left(q^{b-a} t^{2} ; q\right)_{a}(q-t)(t ; q)_{k-1}(t ; q)_{b-a-k}[b-a-k]_{q}\left[\begin{array}{c}
b-a \\
k-1
\end{array}\right]_{q}
$$


(3.15)

$$
\sum_{w \in \mathcal{F}_{2}^{2}} D_{(w, \mu)}(q, t)=(1-t)(t ; q)_{a-1}\left(q^{b-a} t^{2} ; q\right)_{a}(t ; q)_{k-1}(t ; q)_{b-a-k+1}[a]_{q}\left[\begin{array}{c}
b-a \\
k-1
\end{array}\right]_{q},
$$

and

$$
\sum_{w \in \mathcal{F}_{2}^{3}} D_{(w, \mu)}(q, t)
$$

$$
=(1-t)(t ; q)_{a-1}\left(1-q^{b-k-1} t^{2}\right)\left(q^{b-a} t^{2} ; q\right)_{a-1}(t ; q)_{b-a-k}\left(q^{-1} t ; q\right)_{k} q^{k}[a]_{q}\left[\begin{array}{c}
b-a \\
k
\end{array}\right]_{q} \text {. }
$$

We get $\left\langle J_{(b, a)}[X, q, t], s_{(b-k-1, a+k, 1)}\right\rangle$ by simplifying $(3.11)+(3.12)+(3.13)-(3.14)-$ $(3.15)-(3.16)$.

3.3. Rectangular shapes. Here, we consider the case when $\mu=\left(b^{m}\right)$, for $m \geq 2$. We make an observation which enables us to obtain the Schur coefficients indexed by the partition of the form $\left(b-r, b-s, 1^{k}\right)$ of $J_{\left(b^{m}\right)}$ from the Schur coefficients of a similar form in the expansion of $J_{(b, b)}$.

PROPOSITION 3.8. For $m \geq 2$,

$$
\begin{aligned}
\left\langle J_{\left(b^{m}\right)}\right. & \left.(X ; q, t), s_{\left(b-r, b-s, 1^{(r+s+b(m-2))}\right)}\right\rangle \\
\quad= & \left.\left(\prod_{i=1}^{m-2}\left(q^{-b+1} t^{i} ; q\right)_{b} \cdot q^{\left(\begin{array}{c}
b \\
2
\end{array}\right)}\right)\left\langle J_{(b, b)}, s_{\left(b-r, b-s, 1^{r+s}\right)}\right\rangle\right|_{\substack{t \rightarrow t^{m-1} \\
t^{2} \rightarrow t^{m}}},
\end{aligned}
$$

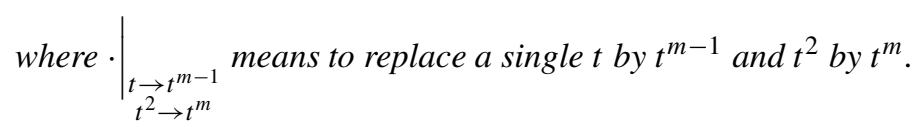

PROOF. Let $\lambda=\left(b-r, b-s, 1^{(r+s+b(m-2))}\right)$. There are two special rim-hook tableaux of shape $\lambda$, of sign +1 and -1 , each. See Figure 3. The special rim-hook tableaux give the following set of fillings

$$
\begin{gathered}
\mathcal{F}_{1}=\{(1,2, \ldots, b-r) \\
\uplus(b-r+1, b-r+2, \ldots, b-r+b-s) \\
\varpi(b m, b m-1, \ldots, b-r+b-s+1)
\end{gathered}
$$

such that $b-r+1$ precedes $b-r$ and $b-r+b-s+1$ precedes $b-r+b-s\}$,

$$
\begin{gathered}
\mathcal{F}_{2}=\{(1,2, \ldots, b-s-1) \amalg(b-s, b-s+1, \ldots, b-r+b-s) \\
\varpi(b m, b m-1, \ldots, b-r+b-s+1)
\end{gathered}
$$

such that $b-s$ precedes $b-s-1$ and $b-r+b-s+1$ precedes $b-r+b-s\}$. 
We want to compute $D_{(w, \mu)}(q, t)$ over the two sets of fillings $\mathcal{F}_{1}$ and $\mathcal{F}_{2}$. Note that the set of numbers $(1,2, \ldots, b-r)$ in $\mathcal{F}_{1}$ and $(1,2, \ldots, b-s-1)$ in $\mathcal{F}_{2}$ should be in the first row of $\mu=\left(b^{m}\right)$ due to the primary condition. Now we claim that the second set of numbers $(b-r+1, \ldots, b-r+b-s)$ in $\mathcal{F}_{1}$ and $(b-s, b-s+1, \ldots, b-r+b-s)$ in $\mathcal{F}_{2}$ should be in the first two rows. Say $b-r+1$ (or $b-s$ in $\mathcal{F}_{2}$ case) comes in higher rows than the first two rows. Then since $b-r+1$ is the smallest number in any triple containing it, inversion and coinversion statistics are zero and hence the cell (say $c$ ) containing $b-r+1$ contributes $t^{\operatorname{leg}(c)+1}-t^{\operatorname{leg}(c)+1}=0$ to $D_{(w, \mu)}(q, t)$. Hence, to fill $\mu$ with $\mathcal{F}_{1}$ or $\mathcal{F}_{2}$, we fill the top $(m-2)$ rows with $b m, b m-1, \ldots$ in decreasing order, from left to right, starting from the top row. These cells contribute $\left(\prod_{i=1}^{m-2}\left(q^{-b+1} t^{i} ; q\right)_{b} \cdot q^{\left(\begin{array}{c}b \\ 2\end{array}\right)}\right)$ to $D_{(w, \mu)}(q, t)$. The rest of the fillings in the remaining two rows will be the same to that we consider to compute $\left\langle J_{(b, b)}, s_{\left(b-r, b-s, 1^{r+s}\right)}\right\rangle$. The replacements of $t$ and $t^{2}$ are due to the difference of the leg lengths.

When $\mu=(b, b)$, we have an explicit expression for $\left\langle J_{(b, b)}, s_{\left(b-r, b-s, 1^{r+s}\right)}\right\rangle$.

Proposition 3.9. For $\mu=(b, b), 0 \leq r \leq s \leq b-1$,

$$
\begin{aligned}
\left\langle J_{(b, b)}\right. & \left.X, q, t), s_{\left(b-r, b-s, 1 r^{r+s}\right)}\right\rangle \\
= & \sum_{k=1}^{r}\left(\left(t ; q^{-1}\right)_{s+k}(t ; q)_{b-s-k}\left(q^{b-s-r-1} t^{2} ; q\right)_{s+1}\left(t^{2} ; q\right)_{b-s-1}\right. \\
& \left.\times q^{r+\left(\begin{array}{c}
s+k \\
2
\end{array}\right)+\left(\begin{array}{c}
r-k+1 \\
2
\end{array}\right)} \frac{[s-r+1]_{q}}{[r]_{q}}\left[\begin{array}{c}
b \\
s+k
\end{array}\right]_{q}\left[\begin{array}{c}
s+k \\
s+1
\end{array}\right]_{q}\left[\begin{array}{c}
s \\
r-k
\end{array}\right]_{q}\right) \\
& +\chi(r+s>b)\left(t ; q^{-1}\right)_{b}\left(t^{2} ; q\right)_{b-s-1}\left(q^{b-r-s-1} t^{2} ; q\right)_{s+1} q^{r+\left(\begin{array}{c}
b \\
2
\end{array}\right)+\left(\begin{array}{c}
r+s-b+1 \\
2
\end{array}\right)} \\
& \times \frac{[s-r+1]_{q}}{[s+1]_{q}}\left[\begin{array}{c}
b \\
r
\end{array}\right]_{q}\left[\begin{array}{c}
r-1 \\
b-s-1
\end{array}\right]_{q}
\end{aligned}
$$

where $\chi(A)=1$ if $A$ is true and 0 otherwise.

PROOF. There are two special rim-hook tableaux of shape $\lambda=\left(b-r, b-s, 1^{r+s}\right)$ as it is shown in Figure 3. The set of fillings of the given descent set corresponding to the special rim-hook tableaux with sign +1 is

$$
\begin{gathered}
\mathcal{F}_{1}=\bigcup_{k=1}^{r}\{(n, n-1, \ldots, n-s-k+1) \amalg(b-r+1, b-r+2, \ldots, n-r-s-k) \| \\
(1,2, \ldots, b-r) \amalg(n-r-s-k+1, n-r-s-k+2, \ldots, n-r-s) \\
\uplus(n-s-k, n-s-k-1, \ldots, n-r-s+1)
\end{gathered}
$$

such that $n-r-s+1$ precedes $n-r-s$ in the bottom row

$$
\cup \chi(r+s>b)\{(n, n-1, \ldots, n-b+1) \|
$$


$[(1,2, \ldots, b-r) \amalg(b-r+1, \ldots, 2 b-r-s) \amalg(b, b-1, \ldots, 2 b-r-s+1)]$

such that $b-r+1$ precedes $b-r$ and $2 b-r-s+1$ precedes $2 b-r-s\}$.

Note that the first union of sets should stop when $k=b-s$ if $r+s>b$. Then by computation, we get

$$
\begin{aligned}
& \sum_{w \in \mathcal{F}_{1}} D_{(w, \mu)}(q, t) \\
& =\sum_{k=1}^{r}\left(\left(t ; q^{-1}\right)_{s+k}(t ; q)_{b-s-k}\left(q^{b-s-r} t^{2} ; q\right)_{r}\left(t^{2} ; q\right)_{b-r} q^{\left(\begin{array}{c}
s+k \\
2
\end{array}\right)+\left(\begin{array}{c}
r-k+1 \\
2
\end{array}\right)}\right. \\
& \left.\times\left[\begin{array}{c}
b-r \\
b-s-k
\end{array}\right]_{q}\left[\begin{array}{l}
r-1 \\
k-1
\end{array}\right]_{q}\left[\begin{array}{l}
b \\
r
\end{array}\right]_{q}\right) \\
& +\chi(r+s>b)\left(t ; q^{-1}\right)_{b}\left(t^{2} ; q\right)_{b-r}\left(q^{b-r-s} t^{2} ; q\right)_{r} q^{\left(\begin{array}{c}
b \\
2
\end{array}\right)+\left(\begin{array}{c}
r+s-b+1 \\
2
\end{array}\right)}\left[\begin{array}{l}
b \\
r
\end{array}\right]_{q}\left[\begin{array}{c}
r-1 \\
b-s-1
\end{array}\right]_{q} \text {. }
\end{aligned}
$$

Similarly, from the tableaux of sign -1 , we have

$$
\mathcal{F}_{2}=\bigcup_{k=1}^{r}\{(n, n-1, \ldots, n-s-k+1) \amalg(b-s, b-s+1, \ldots, n-2 s-k-1) \|
$$

$(1,2, \ldots, b-s-1) ш(n-2 s-k, n-2 s-k+1, \ldots, n-r-s) \amalg$

$$
(n-s-k, n-s-k-1, \ldots, n-r-s+1)
$$

such that $n-r-s+1$ precedes $n-r-s$ in the bottom row

$$
\cup \chi(r+s>b)\{(n, n-1, \ldots, n-b+1) \|
$$

$[(1,2, \ldots, b-s-1) ш(b-s, b-s+1, \ldots, 2 b-r-s) ш(2 b, 2 b-1, \ldots, 2 b-r-s+1)]$

such that $b-s$ precedes $b-s-1$ and $2 b-r-s+1$ precedes $2 b-r-s\}$,

$$
\begin{aligned}
& \sum_{w \in \mathcal{F}_{2}} D_{(w, \mu)}(q, t) \\
&= \sum_{k=1}^{r}\left(\left(q^{-s-k+1} t ; q\right)_{s+k}(t ; q)_{b-s-k}\left(q^{b-s-r} t^{2} ; q\right)_{s+1}\left(t^{2} ; q\right)_{b-s-1} q_{2}^{\left(\begin{array}{c}
s+k \\
2
\end{array}\right)+\left(\begin{array}{c}
r-k+1 \\
2
\end{array}\right)}\right. \\
& \times {\left.\left[\begin{array}{c}
b-s-1 \\
k-1
\end{array}\right]_{q}\left[\begin{array}{c}
s \\
r-k
\end{array}\right]_{q}\left[\begin{array}{c}
b \\
s+1
\end{array}\right]_{q}\right) } \\
&+\chi(r+s>b)\left(q^{-b+1} t ; q\right)_{b}\left(t^{2} ; q\right)_{b-s-1}\left(q^{b-r-s} t^{2} ; q\right)_{s+1} q^{\left(\begin{array}{c}
b \\
2
\end{array}\right)+\left(\begin{array}{c}
r+s-b+1 \\
2
\end{array}\right)}
\end{aligned}
$$




$$
\times\left[\begin{array}{c}
b \\
s+1
\end{array}\right]_{q}\left[\begin{array}{c}
s \\
b-r
\end{array}\right]_{q} .
$$

$\left\langle J_{(b, b)}(X, q, t), s_{\left(b-r, b-s, 1^{r+s}\right)}\right\rangle$ is the result of simplifying (3.18)-(3.19).

Combining Proposition 3.8 and Proposition 3.9 gives the coefficient of the Schur function $s_{\left(b-r, b-s, 1^{(r+s+b(m-2))}\right)}$ in $J_{\left(b^{m}\right)}(X ; q, t)$ for any valid $r, s$ values, $m>2$.

REMARK 3.10. The idea used to prove Proposition 3.8 can be applied to more general situation finding the $s_{\left(b+\alpha-r, b+\beta-s, 1^{(r+s+b(m-2))}\right)}$ coefficient of $J_{\mu}$ when $\mu=\left(b+\alpha, b+\beta, b^{m}\right)$ :

$$
\begin{aligned}
\left\langle J_{\left(b+\alpha, b+\beta, b^{m}\right)}(X ; q, t), s_{\left(b+\alpha-r, b+\beta-s, 1^{(r+s+b m)}\right)}\right\rangle & \\
= & \left.\left(\prod_{i=1}^{m}\left(q^{-b+1} t^{i} ; q\right)_{b} \cdot q^{\left(\begin{array}{c}
b \\
2
\end{array}\right)}\right)\left\langle J_{(b+\alpha, b+\beta)}, s_{\left(b+\alpha-r, b+\beta-s, 1^{r+s}\right)}\right\rangle\right|_{\substack{t \rightarrow t^{m+1} \\
t^{2} \rightarrow t^{m+2}}} .
\end{aligned}
$$

REMARK 3.11. Note the $q, t$-Kostka polynomials $K_{\lambda \mu}(q, t)$ of Macdonald's defined by

$$
J_{\mu}(X ; q, t)=\sum_{\lambda \vdash|\mu|} K_{\lambda \mu}(q, t) s_{\lambda}[X(1-t)],
$$

where the square bracket implies the plethystic substitution. For the details of it, see [Hai99]. When $t=0, s_{\lambda}[X]$ is just the ordinary Schur function $s_{\lambda}(X)$, and by the property of the $q, t$-Kostka polynomials that

$$
K_{\lambda \mu}(q, t)=K_{\lambda^{\prime} \mu^{\prime}}(t, q),
$$

and

$$
K_{\lambda \mu}(0, q)=K_{\lambda \mu}(q)=\sum_{T \in \operatorname{SSYT}(\lambda, \mu)} q^{\operatorname{ch}(T)},
$$

where $K_{\lambda \mu}(q)$ is the Kostka-Foulkes polynomial and $\operatorname{ch}(T)$ is the charge statistic of Lascoux and Schützenberger [LS78], we have

$$
\begin{aligned}
J_{\mu}(X ; q, 0) & =\sum_{\lambda \vdash|\mu|} K_{\lambda \mu}(q, 0) s_{\lambda}[X] \\
& =\sum_{\lambda \vdash|\mu|} K_{\lambda^{\prime} \mu^{\prime}}(0, q) s_{\lambda}(X) \\
& =\sum_{\lambda \vdash|\mu|} K_{\lambda^{\prime} \mu^{\prime}}(q) s_{\lambda}(X) .
\end{aligned}
$$

Hence, we can get some information about the Kostka-Foulkes polynomials from the explicit expressions for the Schur coefficients of $J_{\mu}(X ; q, 0)$. 


\section{References}

[Ass07] SAmi HaYes Assaf, Dual equivalence graphs, ribbon tableaux and Macdonald polynomials, Thesis (Ph.D.)-University of California, Berkeley, ProQuest LLC, Ann Arbor, MI, 2007.

[ELW10] ERIC EgGe, Nicholas A. Loehr and Gregory S. WARrington, From quasisymmetric expansions to Schur expansions via a modified inverse Kostka matrix, European J. Combin. 31 (2010), 2014-2027.

[ER90] ÖMER EĞECIOĞLU and JEFFREY B. REMMEL, A combinatorial interpretation of the inverse Kostka matrix, Linear and Multilinear Algebra 26 (1990), 59-84.

[Ges84] IRA M. Gessel, Multipartite $P$-partitions and inner products of skew Schur functions, Combinatorics and algebra (Boulder, Colo., 1983), Contemp. Math. 34 (1984), 289-317.

[Hag08] JAmES HaGLUnd, The q,t-Catalan numbers and the space of diagonal harmonics, University Lecture Series, vol. 41, American Mathematical Society, 2008.

[Hai99] MARK Haiman, Macdonald polynomials and geometry, New perspectives in algebraic combinatorics (Berkeley, CA, 1996-97), Math. Sci. Res. Inst. Publ., Cambridge Univ. Press 38 (1999), 207-254.

[Hai01] MARK HAIMAN, Hilbert schemes, polygraphs and the Macdonald positivity conjecture, J. Amer. Math. Soc. 14 (2001), no. 4, 941-1006 (electronic).

[HHL05] JAmes Haglund, MARK HAimAn and Nick LoEHR, A combinatorial formula for Macdonald polynomials, J. Amer. Math. Soc. 18 (2005), no. 3, 735-761 (electronic).

[LS78] Alain LascouX and Marcel-Paul SchütZenberger, Sur une conjecture de H. O. Foulkes, C. R. Acad. Sci. Paris Sér. A-B 286 (1978), no. 7, A323-A324.

[Mac95] IAn. G. Macdonald, Symmetric functions and Hall polynomials, second ed., Oxford Mathematical Monographs, The Clarendon Press Oxford University Press, New York, 1995.

[Sta99] Richard P. Stanley, Enumerative combinatorics. Vol. 2, Cambridge Studies in Advanced Mathematics, vol. 62, Cambridge University Press, Cambridge, 1999, With a foreword by Gian-Carlo Rota and appendix 1 by Sergey Fomin.

[Yoo12] MeEsue Yoo, A combinatorial formula for the Schur coefficients of the integral form of the Macdonald polynomials in the two column and certain hook cases, Ann. Comb. 16 (2012), no. 2, 389-410.

Present Address:

FAKULTÄT FÜR MATHEMATIK,

UNIVERSITÄT WIEN,

OSKAR-MORGENSTERN-PLATZ 1,

1090 WiEn, Austria.

e-mail: meesue.yoo@univie.ac.at 